\title{
Accommodative-Convergence Mechanism failure in HIV-Positive Non Presbyopic Patient on Highly Active Anti-Retroviral Therapy: A case report.
}

\author{
Sadiq Hassan ${ }^{1}$, Philips I. Ebisike ${ }^{2}$, Christopher O. Timothy ${ }^{3}$, Basheer A. Z Chedi ${ }^{4}$, \\ Rabi Y. Sani ${ }^{1}$, Saudat G. Habib ${ }^{1}$ \\ 1. Department of Ophthalmology, Faculty of Clinical Sciences, College of Health Sciences, Bayero \\ University Kano/ Aminu Kano Teaching Hospital Kano, Nigeria. \\ 2. Department of Optometry, Faculty of Allied Health Sciences, College of Health Sciences, Bayero \\ University Kano/ Department of Ophthalmology, Aminu Kano Teaching Hospital Kano, Nigeria. \\ 3. Department of Optometry, Faculty of Health Science, College of Medicine and Health Sciences, Abia \\ State University Uturu, Nigeria. \\ 4. Department of Pharmacology and Therapeutics, Faculty of Pharmaceutical Sciences, Bayero University \\ Kano, Nigeria
}

Corresponding Author: Ebisike Philips Ifeanyichukwu | Email: philipsokere@yahoo.com | $+234(0) 7035360969$.

\begin{abstract}
Purpose: Accommodative-convergence mechanism failure could occur in non presbyopic HIV- sero positive patients on Highly Active Anti-Retroviral Therapy (HAART). This could be due to either direct neuronal infection by HIV, pathologic changes of the lens or the ciliary body or adverse effects of some individual drugs constituting the HAART regimen on the cranial nerves which play vital roles in the mechanism of accommodation and convergence.

Case report: This is a case report of an accommodative- convergence mechanism failure in HIV positive non presbyopic 32-year-old male patient that was on HAART for more than five years. He presented with distance visual acuity (VA) of OD: $6{ }_{9}^{+2}$, OS: $6 \%$, and near visual acuity (NVA) of $\mathrm{N}_{24}$ both eyes $3^{\Delta}$ esophoria at distance and $4^{\Delta}$ esophoria at near. Amplitude of accommodation (AA) was 3.50D and accommodative convergence/accommodation (AC/A) ratio was 6/1. Following comprehensive evaluation, his refractive correction was OD: Plano/-0.50DC X $180 \%$ and OS: Plano/-0.50DC X $90 \%{ }_{5}$ at distance with near addition (Add) 2.50D $\mathrm{N}_{5}$. This was prescribed for regular wear in form of D-Top bifocal lens.

Conclusion:These findings showed that HIV sero-positive adults on HAART could develop accommodativeconvergence mechanism failure, which may be characterized by low amplitude of accommodation, receded near point of convergence and high non presbyopic reading addition. These conditions may be under-recognized and need for reading addition of a non presbyopic age is often overlooked.
\end{abstract}

Key Words: Accommodative-Convergence, HIV, Lateral Phoria, Refractive error.

\section{Introduction}

Accommodation refers to a temporary change in the refractive power of the crystalline lens resulting from contraction of the ciliary muscle, thereby altering the location of the point in space optically conjugate with the retina ${ }^{1}$. Clinical measurement of 
the amplitude of accommodation (AA) provides an indication of the maximum accommodative ability. Areduced amplitude may reflect functional difficulty resulting from a failure to initiate or maintain an appropriate accommodative response, uncorrected refractive error (particularly latent hyperopia), or a wide range of systemic conditions ${ }^{2}$. Amplitude of accommodation declines with age. By the fifth decade of life the accommodative amplitude may decline so that the near point of the eye is more remote than the reading distance. When this occurs, the patient is said to have presbyopia ${ }^{2}$.

Accommodation and convergence are inter-related and they develop together so that a single clear image is appreciated. The ratio of accommodativeconvergence(AC)overaccommodation(A)indicates the relationship between the amount of convergence produced by a stimulus to accommodate and the amount of accommodation which produces that convergence ${ }^{3}$. However, Near Point of Convergence (NPC) and AA are linked with the relationship between Accommodation and Convergence known as $\mathrm{AC} / \mathrm{A}$ ratio. The coupling of accommodation and convergence allows clear stable single binocular vision across a range of viewing distances. A change in accommodation is usually accompanied by a change in convergence known as accommodative convergence. When accommodation is exerted the eyes are induced to converge. The amount of accommodative convergence in prism evoked by 1D of accommodation is known as $\mathrm{AC} / \mathrm{A}$ ratio ${ }^{4}$. The AC/A ratio is a useful measure in the diagnosis and management of binocular vision problems like convergence insufficiency ${ }^{5}$. Although it is well known that age affects visual functions, we still have much to learn about the impact of aging on binocular vision. Several studies have provided normative binocular data especially for near vision and for clinical and young populations.

Once presbyopia occurs, those who are emmetropic will need an optical aid for near but not for distance vision; those who have myopia, will find that they see better at near without their distance correction; and those who have hyperopia will find that they may need a correction for both distance and near vision $^{2,6}$. The age-related decline in accommodation occurs almost universally to less than 2 diopters by the time a person reaches 45 to 50 years, by which time most of the population will have noticed a decrease in their ability to focus on close objects and hence require glasses for reading ${ }^{2}$. Accommodation decreases to about 1 diopter at the age of 70 years $^{6}$. In the clinical setting, the AA is commonly measured subjectively, using either the push-up (PU) or minus lens (ML) technique ${ }^{7}$.

The disease burden of human immunodeficiency virus (HIV) is highest in sub-Saharan Africa but there are few studies on the associated neurocognitive disorders in this region ${ }^{8}$.

HIV is a retrovirus that primarily infects components of the human immune system such as $\mathrm{CD}^{+} \mathrm{T}$ cells, macrophages and dendritic cells. It directly and indirectly destroys $\mathrm{CD}^{+} \mathrm{T}$ cells $\mathrm{s}^{9}$. The virus is a member of the genus Lentivirus, part of the family Retroviridae ${ }^{10}$. 
The highly active antiretroviral therapy (HAART) or Antiretroviral therapy (ART) is the combination of several antiretroviral medicines used to slow the rate at which HIV makes copies of itself (multiplies) in the body. A combination of three or more antiretroviral medicines is more effective than using just one medicine (monotherapy) to treat HIV infection ${ }^{11}$.

Study conducted in South Africa shows that HIV and AIDS patients on antiretroviral drugs may have reduced amplitude of accommodation and might experience presbyopia earlier in life than those without HIV due to differences in biometry and optics of crystalline lens between the cases and the controls $^{12}$.

\section{Case Report}

\section{First visit}

A 32-year-old banker reported to the clinic on $16^{\text {th }}$ October 2018, with complaints of difficulty working with his computer, phone or reading the television headlines/subtitles while watching movies or news. He also had double vision when driving for a long distance, pain in the eyes and episodes of blurred vision. There was a history of inability to read small prints for a long while, headaches and tiredness/ heaviness of the eyes after the day's job at the office.

The patient's last eye examination was six years prior to presentation. He tested positive to HIV and had been on Highly Active Anti-Retroviral Therapy (HAART) (Efavirenz, Lamivudine, Tenofovir, Zidovudine, and Nevirapine) for more than five years. His last CD4+ T cell count was 726 cells per cubic millimeter (cells $\left./ \mathrm{mm}^{3}\right)$.

On clinical examination, the patient's presenting Visual Acuity (VA) was OD: $6{ }_{9}^{+2}$, OS: $\%$, Near Visual Acuity (NVA) was $\mathrm{N}_{24}$. The VA with pinhole was OD: $6 / 6+3, \mathrm{OS}: 6 /{ }_{6}^{+1}$. There were no abnormalities seen on anterior and posterior segment examination of the eyes. The cover and uncover test showed $3^{\Delta}$ esophoria at distance and $4^{\Delta}$ esophoria at near. Findings on dry retinoscopy were OD: - 0.25DS /-0.75DC X 180 and OS: - 0.50DS /-1.00DC X 90, and cycloplegic retinoscopy were OD: +0.50DS /-1.00DC X 180 and OS: +0.75DS /-1.25DC X 90, On subjective refraction, he accepted OD: Plano/0.50DC X 180 and OS: Plano/-0.50DC X 90 with a VA of $6 / 5$ in each eye, NVA of $\mathrm{N}_{24}$. He accepted OD: Plano/-0.50DC X 180 and OS: Plano/-0.50DC X 90 binocularly with a near addition of OU: $+2.50 \mathrm{DS}$ $\mathrm{N}_{5}$ which was prescribed in form of D-Top bifocal lens for regular wear.

The Near Point of Convergence (NPC) and Near Point of Accommodation (NPA) were found to be $12 \mathrm{~cm}$ and $25 \mathrm{~cm}$ respectively, AA using minus-lens to blur method was found to be $3.50 \mathrm{D}, \mathrm{AC} / \mathrm{A}$ was 6/1, the Negative Fusional Vergence (NFV) at far and at near was $5^{\Delta}$ Base-In (BI) and $10^{\Delta}$ Base- In (BI) respectively. The Lateral heterophoria tests showed that at distance, Habitual Phoria (HP) was $3^{\Delta}$ esophoria, Induced Phoria (IP) was $2^{\Delta}$ esophoria, while at near, Habitual Phoria (HP) was $7^{\Delta}$ esophoria, Induced Phoria (IP) was $5^{\Delta}$ esophoria. The differential diagnosis considered in this case were presbyopia, external rectus paresis/paralysis, sixth-nerve palsy and pseudo myopia which were ruled out with cycloplegic retinoscopy. Based on the clinical features suggestive of convergence excess

\footnotetext{
Evian. Clive Primary HIV/AIDS care: a practical guide for primary health care personnel in a clinical and supportive setting (Updated 4th ed.). Houghton [South Africa]: Jacana; 2006. p. 29. Charles B. Hicks MD, Jacques W A J. Reeders \& Philip Charles Goodman, ed. Radiology of AIDS. Berlin [u.a.]: Springer; 2001. p. 19.

UNAIDS/World Health Organization. United Nations report on the global AIDS epidemic. 2013; Accessed 2019 Apr 9.

Mathebula SD, Makunyane PS. Loss of amplitude of accommodation in pre-presbyopic HIV and AIDS patients under treatment with antiretrovirals. Afr Vision Eye Health. 2017;76(1): a411.

https://doi.org/10.4102/aveh.v76i1.411
} 
with mixed astigmatism. The optical prescription (D-top bifocal lens) was dispensed to him for three months regular wear. He was counselled and booked for a possible follow-up review after three months.

\section{Follow-up}

During the follow up visit on $23^{\text {rd }}$ of January 2019, he reported that all his symptoms had resolved and he is more comfortable doing his near visual tasks with his glasses. His presenting VA was OD: $6 / 9{ }^{+1}$, OS: $6 / 9$, NVA was $\mathrm{N}_{24}$ pinhole acuity was OD: $6{ }_{6}^{+2}, \mathrm{OS}: 6 / 6$ and the aided VA was OD: $6{ }_{5}$ OS: $6 / 5$, NVA was $\mathrm{N}_{5}$.
With his optical prescription, NPC and NPA were found to be $10 \mathrm{~cm}$ and $13 \mathrm{~cm}, H P$ was $1^{\Delta}$ esophoria, IP was $3^{\Delta}$ exophoria at distance, HP was $4^{\Delta}$ esophoria and $1^{\Delta}$ exophoria for IP at near respectively. The AA using minus-lens to blur method was found to be $3.75 \mathrm{D}, \mathrm{AC} / \mathrm{A}$ was $5 / 1$, the Negative Fusional Vergence (NFV) at far and at near was $3^{\Delta}$ BaseIn (BI) and $8^{\Delta}$ Base- In (BI) respectively. The patient was counselled and encouraged to continue wearing his glasses regularly and return to the clinic for a possible review after one year, if there was no problem before then.

Table 1: Distribution of the clinical variables as determined during patient's examination and follow-up visit.

\begin{tabular}{|c|c|c|}
\hline Tests & First Visit & Follow-up Visit \\
\hline VA & OD: $6 / 9$, OS: $6 / 9$ & OD: $\%{ }_{9}^{+1}$, OS: $\%$ \\
\hline Aided VA & OD: $6 / 5$, OS: $6 / 5$ & OD: $6 / 5$, OS: $6 / 5$ \\
\hline NVA & $\mathbf{N}_{24}$ & $\mathbf{N}_{5}$ \\
\hline Pinhole Acuity & OD: ${ }^{6}{ }_{6}^{+3}, \mathrm{OS}:{ }_{6}{ }_{6}^{+1}$ & OD: ${ }^{6} /_{6}^{+2}, \mathrm{OS}: 6{ }_{6}^{+3}$ \\
\hline Fundoscopy & No Abnormality Detected & No Abnormality Detected \\
\hline Cover and Uncover Test & $\begin{array}{c}3^{\Delta} \text { esophoria at far and } 4^{\Delta} \text { esophoria at } \\
\text { near }\end{array}$ & $\begin{array}{c}2 \Delta \text { esophoria at far and } 3 \Delta \\
\text { esophoria at near }\end{array}$ \\
\hline Lateral Phoria & $\begin{array}{l}\text { At far; HP: } 3^{\Delta} \text { esophoria. } \\
\text { IP: } 2^{\Delta} \text { esophoria. }\end{array}$ & $\begin{array}{l}\text { At far; HP: } 2^{\Delta} \text { esophoria } \\
\text { IP: } 3^{\Delta} \text { exophoria }\end{array}$ \\
\hline & $\begin{array}{l}\text { At near; HP: } 7^{\Delta} \text { esophoria. } \\
\text { IP was } 5^{\Delta} \text { esophoria. }\end{array}$ & $\begin{array}{l}\text { At near; HP: } 6^{\Delta} \text { esophoria } \\
\text { IP: } 1^{\Delta} \text { exophoria }\end{array}$ \\
\hline Dry Refraction & $\begin{array}{c}\text { OD: - 0.25DS /-0.75DC X } 180 \\
\text { OS: - 0.50DS /-1.00DC X } 90\end{array}$ & Same \\
\hline Cycloplegic Refraction & $\begin{aligned} \text { OD: } & +0.50 \mathrm{DS} /-1.00 \mathrm{DC} \times 180 \mathrm{OS}: \\
& +0.75 \mathrm{DS} /-1.25 \mathrm{DC} \times 90\end{aligned}$ & Same \\
\hline \multirow[t]{2}{*}{ Subjective Refraction } & $\begin{array}{c}\text { OD: Plano/-0.50DC X } 180 \text { Add } \\
\text { 2.50D }\end{array}$ & Same \\
\hline & OS: Plano/-0.50DC X 90 Add 2.50D & Same \\
\hline NPC & $12 \mathrm{~cm}$ & $10 \mathrm{~cm}$ \\
\hline NPA & $25 \mathrm{~cm}$ & $13 \mathrm{~cm}$ \\
\hline NFV & $5^{\Delta} \mathrm{BI}$ at far, $10^{\Delta} \mathrm{BI}$ at near & $3^{\Delta} \mathrm{BI}$ at far, $8^{\Delta} \mathrm{BI}$ at near \\
\hline AA & $3.50 \mathrm{D}$ & $3.75 \mathrm{D}$ \\
\hline AC/A Ratio & $6 / 1$ & $5 / 1$ \\
\hline Diagnosis & $\begin{array}{c}\text { Convergence Excess with Mixed } \\
\text { Astigmatism }\end{array}$ & \\
\hline
\end{tabular}

OD: Right eye, OS: Left eye, HP: Habitual Phoria, IP: Induced Phoria, NFV: Negative Fusional Vergence, NPC: Near Point of Convergence, NPA: Near Point of Accommodation, AA: Amplitude of Accommodation, AC/A Ratio: Accommodative- Convergence per Accommodation Ratio, VA: Visual Acuity and NVA: Near Visual Acuity. 


\section{Discussion}

Highly active antiretroviral therapy is the combination of several antiretroviral medicines used to slow the rate at which HIV multiplies in the body. A combination of three or more antiretroviral medicines is more effective than using just one medicine (monotherapy) to treat HIV infection ${ }^{11}$.

Low amplitude of accommodation may lead to accommodative insufficiency which are often seen in both presbyopia and non-presbyopic age, and it has been reported that HIV-sero positive patients exhibit failed accommodation ${ }^{13}$. Thierfelder et al. reported that reduced amplitude of accommodation is an early ophthalmological symptom of HIV infected patients; they stated that the possible causes may be either direct neuronal infection by HIV 1, pathologic changes of the lens or the ciliary body. But they failed to find any relationship between accommodative failure and CD4+ count ${ }^{14}$.

Neeta and Radhakrishnan in their study identified accommodative failure in a significant proportion of HIV-positive patients on anti-retroviral therapy (ART) aged between 35 and 45years. They also discovered that this problem may be underrecognized and need for near correction for the pre presbyopic age is often overlooked. According to them, Accommodation failure was not related to CD4 count or current ART regimen being used ${ }^{15}$. Amplitude of accommodation as one of the visual functions were also found to be significantly smaller in the HIV-positive group compared with controls for age groups 25-29, but not in the older group. Thirty percent of patients aged between 25 and 34 years had reduced amplitude of accommodation below age-expected norms. Accommodative failure was also found not to be related to current or lowest CD4 count, viral load or specific antiretroviral therapies ${ }^{16}$.

The accommodative- convergence mechanism failure among HIV- sero positive on HAART may occur due to either direct neuronal infection by HIV 1, pathologic changes of the lens or the ciliary body or adverse effects of some individual drugs constituting HAART regimen on the cranial nerves such as oculomotor nerve which play vital roles in the mechanism of accommodation and convergence ${ }^{17}$. Since the case report presented above were on firstand second-line HAART regimen which contain zidovudine, Efavirenz and Lamivudine, Tenofovir and Atazanavir, these drugs may account for the low amplitude of accommodation, receded near point of convergence and high presbyopic reading addition found. As Westcott et al, discussed, it remains a possibility that accommodative impairment in HIV positive patients may arise secondary to changes in the lens resistance to deformation, as a consequence of either age-accelerated changes or pathological change. They also reported that in normal subjects there is evidence that the decline in ciliary body contractility is only minimal before the age of 4550 years. In HIV-positive patients it is possible that impairment of the ciliary body muscle function occurs, as a consequence of either direct changes to the muscle or parasympathetic neuropathy. The causal role of a parasympathetic neuropathy is a possibility as several studies have shown that autonomic neuropathy is frequently found early in the course of HIV infection ${ }^{16}$.

However, the combined individual adverse drug reaction of HAART among the regimen may have also contributed in the reported failed or poor amplitude of accommodation, abnormal high presbyopic reading addition and abnormal receded near point of convergence in HIV/AIDS patients on HAART.As reported by Maschke et al, that the currently used highly active antiretroviral therapy (HAART) for the treatment of HIV infection is associated with long term side effects. According to them, Nucleoside reverse transcriptase inhibitors

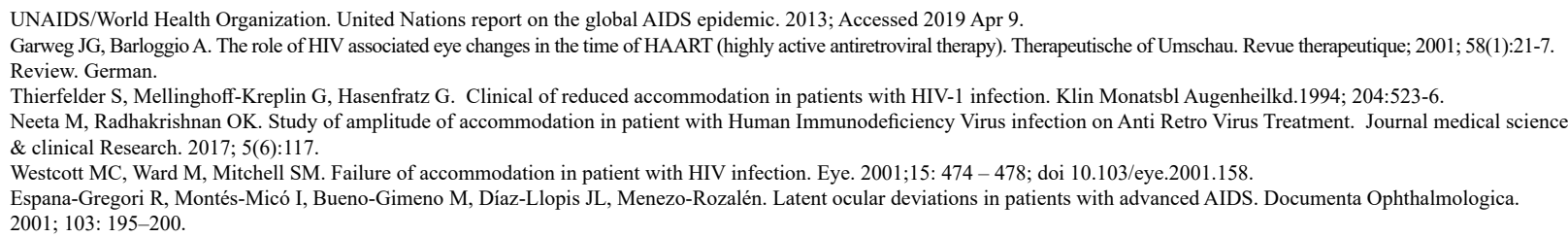


(NRTIs) may cause mitochondrial toxicity, leading to distal symmetric polyneuropathy (DSP). They also reported that nearly one-third of the patients treated with Nucleoside reverse transcriptase inhibitors (NRTIs) experience peripheral neuropathic side effect $^{18}$.

The works of Mackey, and Shaikh et al, as reported by Sui-Yi et al, also supported that drug resistance, adverse side effect and drug-drug interaction have been universal problem with the application of HAART for HIV infected patients. They stated that the toxicity of nucleoside reverse transcriptase inhibitor (NRTIs) therapy e.g. Zidovudine, may cause potential inhibition of host mitochondrial DNA polymerase and the long time use of Zidovudine may also account for the late complication of optic neuropathy ${ }^{19-21}$.

Also, Brinkman et al, supported this and concluded that Mitochondrial toxicity is a clearly recognized adverse effect of NRTI and that the clinical features of this toxicity, which can be both reversible and irreversible, vary inter-individually and between several tissues ${ }^{22}$. Moreover, they also discovered that Zidovudine and protease inhibitors (Is) used alone can induced endothelial cell proliferation, causing a dysregulation of angiogenesis, which makes HIVpositive patients more prone to hemangiomas ${ }^{22}$. The results of this case report also correlated with their findings, in that the aforementioned patient was on HAART regimen, he had an abnormal near point of convergence, amplitude of accommodation and high presbyopic reading addition, which may be related to the neuropathies of the cranial nerves such as optic nerve, oculomotor nerve and abducent nerve etc. These cranial nerves play an important role in the innervation of the accommodativeconvergence mechanism.

Espana-Gregori et al, reported that the horizontal latent ocular deviation at near and at distance in advanced AIDS patients showed lower values than the expected. The AC/A relationship also were lower. The results obtained in this study indicate that AIDS patients suffer a convergence excess, which could add to other visual complaints such as blurred vision, photophobia, Nyctalopia and reading difficulty at near. The findings also suggest that there is a variation of the deviation angles in AIDS patients, giving lower than expected values at near and at distance, due to a possible oculomotor muscle alteration produced in relation to the degree of HIV infection ${ }^{17}$. In agreement with their findings, our present study also found that the lateral latent ocular deviation for habitual and induced phorias at near and distance vision in HIV- sero positive population on HAART were abnormal compared with the expected normal range with their age. The patient under review had esophoria at near and distance for habitual phoria and esophoria at near and distance for induced phoria.

Similar to the findings of Espana-Gregori et al., the results from this study showed that HIV-sero positive patients on HAART exhibit convergence excess which could add to other visual complaints such as blurred vision, photophobia, headache, diplopia and difficulty reading tiny prints at near distance ${ }^{17}$. These findings also confirmed that the different deviation angles that are subtended by their visual axes also exist and the etiology of these binocular vision anomalies among the HIV-sero positive patients on HAART may be due to their receded near point of convergence, low amplitude of accommodation with high presbyopic reading addition, autonomic dysfunction associated with the innervation of the accommodative-convergence mechanism, pathological changes due to the HIV virus or the effect and duration of HAART. However, we recommend further studies that will entail large population samples, so as to validate these findings and determine its prevalence rate within various localities.

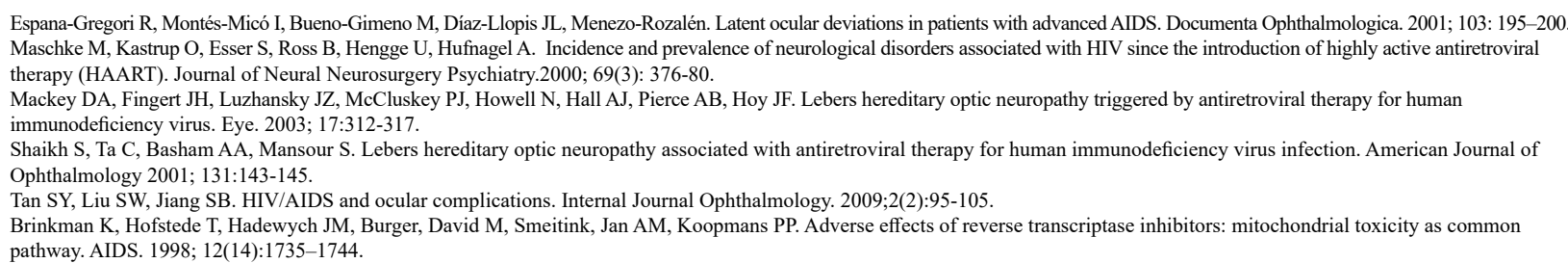




\section{Conclusion}

The findings of this study, showed that HIV sero-positive adults on HAART were more likely to develop at least one or more form of accommodative-convergence mechanism dysfunction and that low amplitude of accommodation, high non presbyopic reading addition and binocular vision anomalies may be associated with duration on HAART. These abnormalities of accommodative- convergence mechanism may lead to frequent change of spectacle correction or spectacle intolerance among HIV sero-positive non presbyopic patients on HAART, as these conditions are under recognized in most cases and the need for reading addition on a non presbyopic patients are often overlooked by many clinicians. Therefore, clinicians should pay more attention to these similar complains, clinical test results and patients from this class of population. 